\title{
AVIAN-INSPIRED GRASPING FOR QUADROTOR MICRO UAVS
}

\author{
Justin Thomas \\ GRASP Lab \\ University of Pennsylvania \\ Philadelphia, Pennsylvania \\ Email: jut@seas.upenn.edu

\section{Koushil Sreenath} \\ GRASP Lab \\ University of Pennsylvania \\ Philadelphia, Pennsylvania
}

\author{
Joe Polin \\ GRASP Lab \\ University of Pennsylvania \\ Philadelphia, Pennsylvania
}

\author{
Vijay Kumar \\ GRASP Lab \\ University of Pennsylvania \\ Philadelphia, Pennsylvania
}

\section{ABSTRACT}

Micro Unmanned Aerial Vehicles (MAVs) have been used in a wide range of applications [1, 2, 3]. However, there are few papers addressing high-speed grasping and transportation of payloads using MAVs. Drawing inspiration from aerial hunting by birds of prey, we design and equip a quadrotor MAV with an actuated appendage enabling grasping and object retrieval at high speeds. We develop a nonlinear dynamic model of the system, demonstrate that the system is differentially flat, plan dynamic trajectories using the flatness property, and present experimental results with pick-up velocities at $2 \mathrm{~m} / \mathrm{s}$ ( 6 body lengths / second) and $3 \mathrm{~m} / \mathrm{s}$ ( 9 body lengths / second). Finally, the experimental results are compared with observations derived from video footage of a bald eagle swooping down and snatching a fish out of water.

\section{INTRODUCTION}

Predatory birds have the ability to swiftly swoop down from great heights and grasp prey, with high rates of success, from the ground, water, and air while flying at high speeds [5]. Although recent years have seen improvement in the capabilities of Micro Unmanned Aerial Vehicles (MAVs) [6, 7], such dynamic aerial manipulation, common in nature, has not been achieved using MAVs. The present state of the art in aerial manipulation ranges

This work is supported in part by ONR Grant N00014-07-1-0829 and ARL Grant W911NF-08-2-0004.

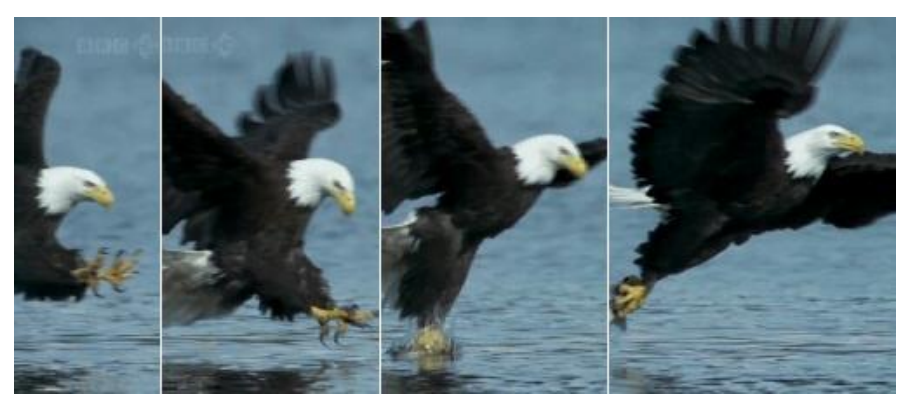

FIGURE 1. Video frames of an eagle grasping prey [4].

from using grippers for construction [1] to cable-suspended loads for dynamic transportation [2]. Acquiring, transporting and deploying payloads while maintaining a significant velocity are important since they would save MAVs time and energy by minimizing required flight time. For example, high-speed grasping could be used in rescue operations where speed and time are critical, and in operations requiring a MAV to quickly swoop down and pick up an object of interest. Additionally, the dynamic grasping functionality could be extended to achieve perching, which could be used to quickly escape high winds, achieve immediate silence in stealth operations, and improve mission duration by reducing hover time.

With the recent increase in such MAV applications, there has also been a rising need for articulated appendages capable of interacting with the environment. Dollar et al. developed fin- 
gers that passively conformed to a wide range of object shapes [8]; Doyle et al. developed a passively actuated gripper to facilitate perching [9]; Lindsey et al. designed a servo-driven claw to transport plastic construction beams [1]; and Mellinger et al. utilized a gripper with fish hooks to pierce its targets [10]. Though these grippers vary in method and application, they suffer from a common limitation; in order to be effective, the vehicle must make an approach perpendicular to the plane of the target and the approach velocity must be close to zero when grasping. The ingressive gripper in [11] was able to perch with more aggressive trajectories by triggering a spring-loaded claw that would engage upon contact, but still needed to contact the target surface with a normal velocity.

Video analysis of birds of prey, such as the bald eagle (Haliaeetus leucocephalus) shown in Fig. 1, reveal that an eagle sweeps its legs and claws backwards during its capture phase, thereby reducing the relative velocity between the claws of the predator and the prey [4]. This allows the bird, without slowing down, to have a near-zero relative velocity of the claw while grasping the prey. This strategy provides a high rate of success in grasping prey, even though most fish can maneuver quickly out of harm's way if they can detect the predator far enough in advance. We draw inspiration from this method to enable highspeed aerial grasping and manipulation for MAVs.

The rest of the paper is organized as follows. We first present a novel gripper design capable of reducing the velocity of the claw relative to the target. Next, we present the dynamic model of the quadrotor MAV equipped with the gripper, and we demonstrate that the system is differentially flat. Following this, we present a trajectory generation method based on the flat outputs and provide an overview of the controllers. The next section presents experimental results of high-speed grasping at $2 \mathrm{~m} / \mathrm{s}$ and $3 \mathrm{~m} / \mathrm{s}$. In addition, we provide a nondimensional comparison between a MAV claw trajectory and a sample avian claw trajectory. Finally, we present concluding remarks with thoughts for future work.

\section{DESIGN OF AN ARTICULATED GRIPPER}

Gripper design is critical for high-speed aerial manipulation. A primary goal of a successful gripper is to enable MAVs to acquire payloads while moving at significant relative velocities. A secondary goal is to enable the ability to perch by compliantly grasping objects of arbitrary shape or features such as tree branches or roof tops that are available in typical urban environments.

An initial gripper design resembled a two-pronged fork that interfaces with a plastic ball fixed to the payload, as seen in Fig. 2. The fork is 3D printed from Acrylonitrile Butadiene Styrene (ABS) and is designed to guide the ball into a spherical recess where it remains secure. A quadrotor equipped with this claw can acquire payloads at relative speeds up to $1 \mathrm{~m} / \mathrm{s}$. To release
TABLE 1. Nomenclature

- $q \quad$ Subscript " $\mathrm{q}$ " denotes the Quadrotor

- $g \quad$ Subscript " $\mathrm{g}$ " denotes the Gripper

is Subscript "s" denotes the coupled system

-c Subscript "c" denotes a commanded signal

$\stackrel{d}{d} \quad$ Superscript "d" denotes desired or feed-forward

$\mathbf{r}, \in \mathbb{R}^{3}$ Position of a Center of Mass (CoM)

$\mathbf{b}_{i} \in \mathbb{R}^{3}$ The $i^{t h}$ body-fixed basis vector

$\mathbf{q} \in \mathbb{R}^{4}$ Vector of generalized coordinates

$\mathbf{y} \in \mathbb{R}^{3}$ Flat Outputs

$\theta \in \mathbb{R} \quad$ Attitude of the Quadrotor

$\Omega \in \mathbb{R}^{3}$ Angular velocity of the Quadrotor about $\mathbf{b}_{2}$

$\beta \in \mathbb{R} \quad$ Angle of the Gripper relative to the horizonatal

$m . \in \mathbb{R} \quad$ Mass of a system

$J, \in \mathbb{R} \quad$ Angular inertia of a system

$u_{1} \in \mathbb{R} \quad$ Total thrust along the $\mathbf{b}_{1}$ axis

$u_{3} \in \mathbb{R} \quad$ Moment along the $\mathbf{b}_{2}$ axis from differential thrusts

$\tau \in \mathbb{R} \quad$ Arm actuator torque along the $\mathbf{b}_{2}$ axis

$L_{g} \in \mathbb{R} \quad$ Length between Quadrotor and Gripper CoMs

$x^{\star} \in \mathbb{R} \quad$ Non-dimensionalized $x$ position of the claw

$z^{\star} \in \mathbb{R} \quad$ Non-dimensionalized $z$ position of the claw

$t^{\star} \in \mathbb{R} \quad$ Non-dimensionalized time

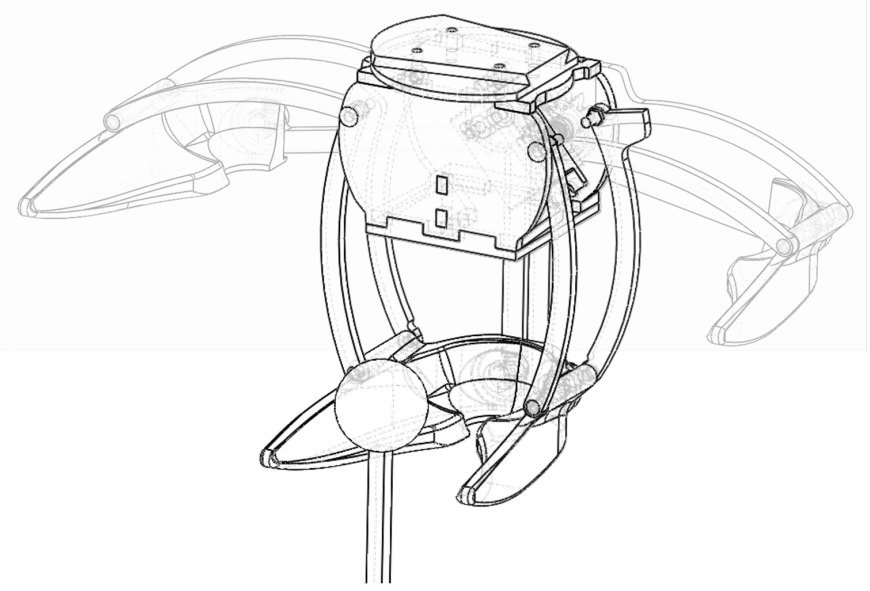

FIGURE 2. An early prototype gripper that could be used with spherical targets. The gripper is shown in its open (shaded) and closed (solid) configurations.

the payload, the fork can be separated by a mini servo motor. However, this claw requires specialized fixtures on the payload and is incapable of grasping objects at higher speeds due to large relative velocities between the claw and the target.

To enable more adaptable grippers, the finger design used in [12] is adopted for the quadrotor platform. A similar mechanism was studied in [13]. As a result of this actuation design, the three fingers conform to the object while collectively driven by a sin- 


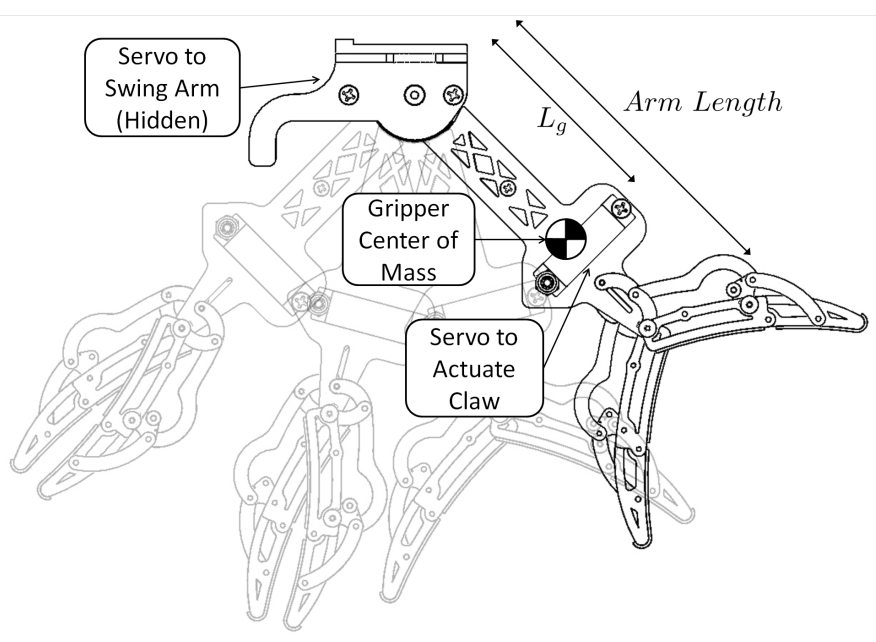

FIGURE 3. The gripper arm in motion as the claw is grasping. The shaded projections demonstrate the motion as the arm swings about the axis pointed into the page $\left(\mathbf{b}_{2}\right)$.

gle servo motor. The fingers are constructed from laser-cut ABS and covered with Dycem, a high-friction rubber that is used to improve grip. Although this design facilitates the grasping of arbitrary object shapes, the fingers alone cannot close fast enough to capture payloads if the quadrotor is in motion.

To reduce the relative speed between the gripper and the target, we draw inspiration from the way an eagle sweeps its legs backward just prior to grasping. In particular, the actuated gripper is mounted on a $10.5 \mathrm{~cm}$ rotating arm. The arm, also composed of laser-cut ABS, pivots directly below the quadrotor's center of mass and is actuated by a mini servo motor. When the arm rotates, the gripper experiences a velocity which reduces the relative velocity between itself and the payload during acquisition. See Fig. 3 for a time-lapse visualization of the motion.

As we will show later, this gripper design satisfies our goals of compliantly grasping arbitrarily shaped objects and enabling high-speed grasping. The gripper can be further improved by leveraging shape deposition manufacturing (SDM) for fabricating light-weight fingers [14], which will decrease inertia and permit acquisition at even larger velocities.

\section{DYNAMIC MODEL AND DIFFERENTIAL FLATNESS}

We develop a dynamic model for a quadrotor MAV equipped with an articulated gripper (henceforth called the coupled system). The dynamics of a quadrotor platform are well understood $[15,16]$, and involve a net thrust, $u_{1}$, in the direction perpendicular to the plane of the body and moments $u_{2}, u_{3}$, and $u_{4}$ acting along three body-fixed axes, $\mathbf{b}_{1}, \mathbf{b}_{2}$, and $\mathbf{b}_{3}$, respectively. In this paper, we adopt a planar version of this model for two reasons. First, in most examples of avian grasping and perching, the sig-

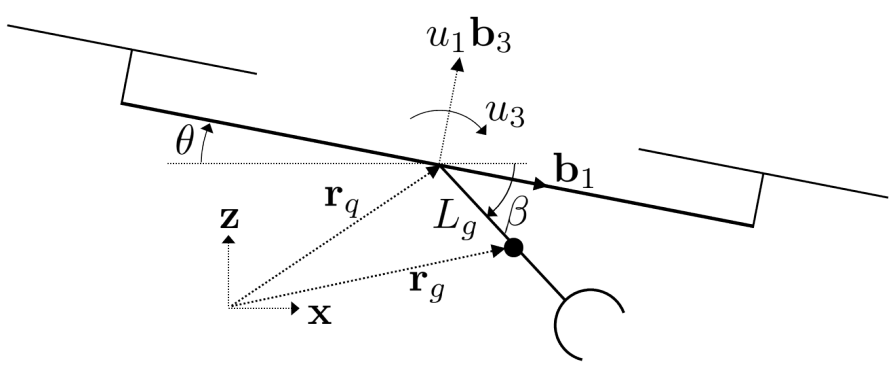

FIGURE 4. The quadrotor has control inputs $u_{1}$ in the $\mathbf{b}_{3}$ direction and $u_{3}$ as a moment about the axis into the page $\left(\mathbf{b}_{2}\right)$. The gripper forms the angle $\beta$ with the horizontal and its center of mass is located a distance $L_{g}$ away from the quadrotor's center of mass.

nificant movements are limited to the sagittal plane of the bird. Indeed, most examples of claws and feet seen in nature have an axis of symmetry. Second, it is difficult to achieve high speed grasping without specifying a plane of approach. Note that most previous work requires the approach to be restricted to a single dimension, instead of two. Thus, we develop a simplified dynamic model, in which we only consider the motion in the $x-z$ plane with two inputs, $u_{1}$ and $u_{3}$. See Fig. 4 for a visualization.

The angle of the gripper relative to the horizontal $(x-$ axis $)$ is defined as $\beta$, as displayed in Fig. 4 , and the attitude of the vehicle is defined by $\theta$ such that the angle between the quadrotor and the gripper is $\gamma=\beta-\theta$. Further, the masses of the quadrotor and gripper are defined as $m_{q}$ and $m_{g}$, respectively, while the moments of inertia about the center of mass of the planar quadrotor and gripper are defined as $J_{q}$ and $J_{g}$, respectively. The axis of rotation for the gripper is assumed to be at the quadrotor's center of mass so that the fixed distance $L_{g}$ denotes the length from the gripper's center of mass to the quadrotor's center of mass. We express the position vector of the quadrotor and gripper as $\mathbf{r}_{q}=\left[\begin{array}{lll}x_{q} & 0 & z_{q}\end{array}\right]^{T}$ and $\mathbf{r}_{g}=\left[\begin{array}{lll}x_{g} & 0 & z_{g}\end{array}\right]^{T}$, respectively. See Table 1 for a summary of the nomenclature.

The position of the gripper is entirely determined from the position of the quadrotor and the angle of the gripper through

$$
\mathbf{r}_{g}=\mathbf{r}_{q}+L_{g}\left[\begin{array}{c}
\cos (\beta) \\
0 \\
-\sin (\beta)
\end{array}\right]
$$

Furthermore, higher-order time-derivatives of the gripper position can be expressed as functions of the position of the quadrotor, the angle of the gripper, and their higher-order derivatives. 


\section{Dynamics}

The dynamics of the coupled system are determined using Lagrangian mechanics where the potential energy is

$$
V=m_{q} g z_{q}+m_{g} g z_{g},
$$

and the kinetic energy is

$$
T=\frac{1}{2}\left(m_{g}\left\|\dot{\mathbf{r}}_{g}\right\|_{2}^{2}+m_{q}\left\|\dot{\mathbf{r}}_{q}\right\|_{2}^{2}+J_{g} \omega_{g}^{2}+J_{q} \omega_{q}^{2}\right) .
$$

Then, $\mathbf{q}=\left[x_{q} z_{q} \theta \beta\right]^{T}$ is the vector of generalized coordinates so that the corresponding vector of generalized forces and moments is

$$
\mathbf{F}=\left[\begin{array}{c}
u_{1} \sin (\theta) \\
u_{1} \cos (\theta) \\
u_{3}-\tau \\
\tau
\end{array}\right]
$$

where $\tau$ is the actuator torque on the gripper arm. The dynamics are determined using the Euler-Lagrange equations so that

$$
\ddot{\mathbf{q}}=D^{-1}(\mathbf{F}-C \dot{\mathbf{q}}-G)
$$

where the matrices $D, C$, and $G$ are

$$
\begin{aligned}
& D=\left[\begin{array}{cccc}
m_{g}+m_{q} & 0 & 0 & -L_{g} m_{g} s(\beta) \\
0 & m_{g}+m_{q} & 0 & -L_{g} m_{g} c(\beta) \\
0 & 0 & J_{q} & 0 \\
-L_{g} m_{g} s(\beta) & -L_{g} m_{g} c(\beta) & 0 & J_{g}+L_{g}^{2} m_{g}
\end{array}\right], \\
& C=\left[\begin{array}{cccc}
0 & 0 & 0 & -L_{g} m_{g} c(\beta) \dot{\beta} \\
0 & 0 & 0 & L_{g} m_{g} s(\beta) \dot{\beta} \\
0 & 0 & 0 & 0 \\
0 & 0 & 0 & 0
\end{array}\right], \\
& G=\left[\begin{array}{c}
0 \\
g\left(m_{g}+m_{q}\right) \\
0 \\
-g L_{g} m_{g} c(\beta)
\end{array}\right]
\end{aligned}
$$

with $s(\beta)=\sin (\beta)$ and $c(\beta)=\cos (\beta)$.

\section{Differential Flatness}

The dynamic model serves for validation of controllers in simulation. However, to enable planning high-speed dynamic trajectories for aerial grasping, we will demonstrate that the system under consideration is differentially flat $[17,18]$. Differential flatness has been used to plan aggressive trajectories for quadrotor systems [16], and we will take a similar approach. Showing that the system is differentially flat and identifying the flat outputs allows a simplified approach to plan trajectories that minimize control inputs while incorporating dynamic constraints

A system is differentially flat if there exists a change of coordinates which allows the state, $(\mathbf{q}, \dot{\mathbf{q}})$, and control inputs, $\mathbf{u}$, to be written as functions of the flat outputs and their derivatives $\left(y_{i}, \dot{y}_{i}, \ddot{y}_{i}, \ldots\right)$ [18]. Additionally, we require that the flat outputs are functions of the state and the control inputs [18]. If the change of coordinates is a diffeomorphism, we can plan trajectories using the flat outputs and their derivatives in the flat space since there is a unique mapping to the full state space of the dynamic system.

The coupled system comprising of the quadrotor and the actuated gripper, whose dynamics is given by (5), is differentially flat with a set of flat outputs given by (see the Appendix for details)

$$
\mathbf{y}=\left[x_{q} z_{q} \beta\right]^{T} .
$$

Consequently, any sufficiently smooth trajectory in the space of flat outputs is automatically guaranteed to satisfy the equations of motion. Further, we see that the control inputs to the system are functions of the snap $\left(\mathbf{y}^{(4)}\right)$ of the trajectories (see the Appendix). Thus, we require that trajectories planned in the flat space be continuous in position $(\mathbf{y})$, velocity $(\dot{\mathbf{y}})$, acceleration $(\ddot{\mathbf{y}})$, and jerk $(\dddot{\mathbf{y}})$.

\section{TRAJECTORY GENERATION AND CONTROL DESIGN}

From the previous section, further examination of the control inputs reveals that the snap of the position of the quadrotor appears in the $u_{3}$ term through $\ddot{\theta}$. In addition, $\beta^{(4)}$ appears in $u_{3}$ through the $\mathbf{r}_{s}^{(4)}$ term in $\ddot{\theta}$. Then, to minimize the norm of the input vector, it is necessary to minimize the following cost functional constructed from the snap of the trajectory.

$$
\mathscr{J}_{i}=\int_{t_{0}}^{t_{f}}\left\|y_{i}^{(4)}(t)\right\|^{2} d t \quad \text { for } \quad i=1,2,3
$$

Accordingly, we consider minimum-snap trajectories, which can be formulated as a Quadratic Program (QP) and solved numerically, as in [16]. However, we have now added a dimension for $\beta$. 
Although we have a method to generate trajectories for the quadrotor, we do not have a definitive way to determine the constraints for the trajectory. For this, we take inspiration from nature and analyze video footage of an eagle grasping a fish out of water. The segment of video used is from a static viewpoint at an unknown distance and unknown time-scale (the video segment is in slow motion). The extracted trajectory will be compared later in a following section.

The trajectories used for experimentation are constrained by position at the start and finish where the higher derivatives are zero at the desired start and goal locations. The position at pickup is constrained such that the gripper is oriented vertically when grasping the target, but the velocity, acceleration, and jerk of the quadrotor are free and required to be continuous. A fully-defined trajectory was planned for the $x$ and $z$ positions of the quadrotor. In order to ensure a successful grasp, the gripper must be pointed directly at the target during approach. To achieve this, position constraints were placed on the $\beta$ trajectory prior and up to the pickup time so that the gripper would be pointed directly at the target. See Fig. 5 and Fig. 6 for a desired and experimental trajectory of the position of the quadrotor and the gripper angle, respectively.

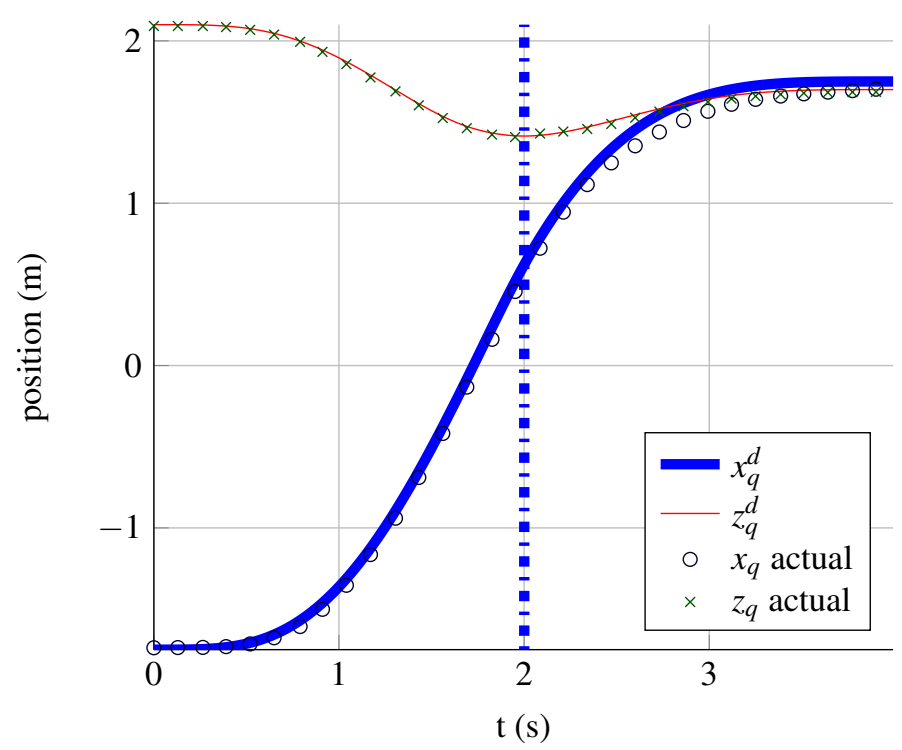

FIGURE 5. Desired quadrotor position trajectories overlayed with experimental results. The planned pickup time is $t=2 \mathrm{~s}$.

Next, we briefly present the controller that drives the quadrotor and gripper system along the desired trajectory. The quadrotor controller has an outer position control loop running at $100 \mathrm{~Hz}$ which generates desired attitudes and feedforward control inputs.

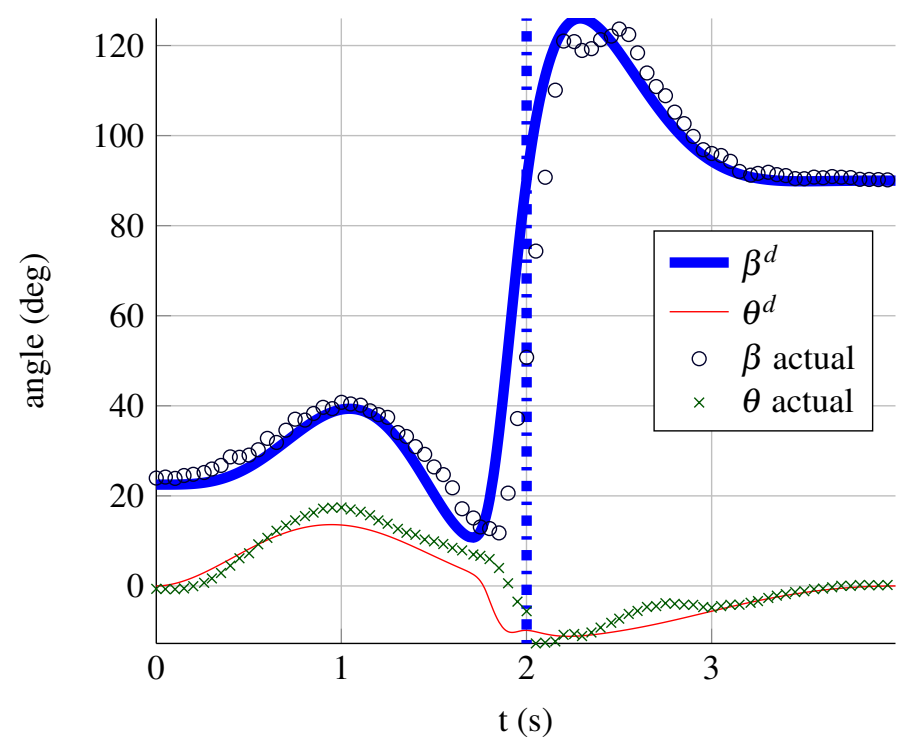

FIGURE 6. Desired $\beta$ and $\theta$ trajectories overlayed with experimenatl results. The planned pickup time is $t=2 s$.

The commanded thrust is

$$
u_{1 c}=k_{p z}\left(z_{q}^{d}-z_{q}\right)+k_{d z}\left(\dot{z}_{q}^{d}-\dot{z}_{q}\right)+u_{1}^{d}
$$

where $k_{p z}$ and $k_{d z}$ are proportional and derivative gains, respectively. The desired values of various variables, denoted with a superscript "d", are computed using the flatness property. A $1 \mathrm{Khz}$ inner-loop attitude controller on-board the quadrotor is used to drive the robot to the desired attitude. The commanded moment is

$$
u_{3 c}=k_{p \theta}\left(\theta_{c}-\theta\right)+k_{d \theta}\left(\dot{\theta}^{d}-\dot{\theta}\right)+u_{3}^{d}
$$

where $\dot{\theta}^{d}$ is the nominal angular rate, $u_{3}^{d}$ is the feed-forward moment, $k_{p \theta}$ is a proportional gain, and $k_{d \theta}$ is a derivative gain. $\theta_{c}$ is the command from the outer loop determined by

$$
\theta_{c}=\sin ^{-1}\left(k_{p x}\left(x_{q}^{d}-x_{q}\right)+k_{d x}\left(\dot{x}_{q}^{d}-\dot{x}_{q}\right)\right)+\theta^{d}
$$

where $k_{p x}$ is a proportional gain and $k_{d x}$ is a derivative gain. The control design is similar to the quadrotor hover controller in [6] and the feedforward control input serves to compensate for the motion of the gripper. The state of the quadrotor is observed using VICON [19] and feedforward control inputs are supplied to the control loops as displayed in Fig. 7. 


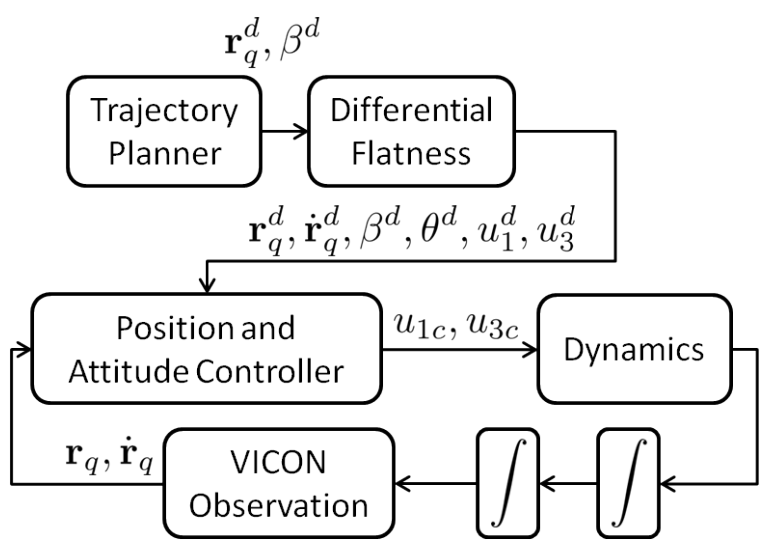

FIGURE 7. A block diagram of the controller used for experiments. A superscript "d" denotes a desired or nominal value (computed using the flatness property).

\section{RESULTS}

We demonstrate experimental results on a $500 \mathrm{gm}$ Asctec Hummingbird quadrotor [20] equipped with a 158 gm gripper. The experiments utilize the GRASP Multiple Micro UAV Testbed [15] and leverage a motion capture system to accurately determine the state of the quadrotor [19]. A $27 \mathrm{gm}$ cylindrical target was tracked using VICON [19].

The controller presented in the earlier section, that combines feedforward control inputs and a simple feedback controller on the quadrotor, was used in experiments, and the gripper claw was commanded to close slightly before the pickup time. With this setup, the quadrotor grasped the target while moving at $2 \mathrm{~m} / \mathrm{s}$ with a success rate of $100 \%$ out of 5 attempts. Position errors for those trajectories are presented in Fig. 8. The quadrotor was able to successfully grasp the target at speeds up to $3 \mathrm{~m} / \mathrm{s}$, or 9 body lengths / second (Fig. 11).

\section{Avian Comparison}

In assessing the success of our results, it is appropriate to use the eagle's performance as a standard of comparison. We desire the same result that the eagle achieves, and therefore expect to match the bird closely.

The footage of the eagle is slowed by an unknown factor resulting in an unknown time scale. The length scale is also impossible to extract accurately. However, it is still meaningful to compare the two nondimensionalized sets of trajectories. We nondimensionalize the trajectories using the following relationships:

$$
x^{\star}=\frac{x}{L}, \quad z^{\star}=\frac{z}{L}, \quad t^{\star}=\frac{t v_{p}}{L}
$$

where $v_{p}$ is the body velocity at pickup and $L$ is the length from
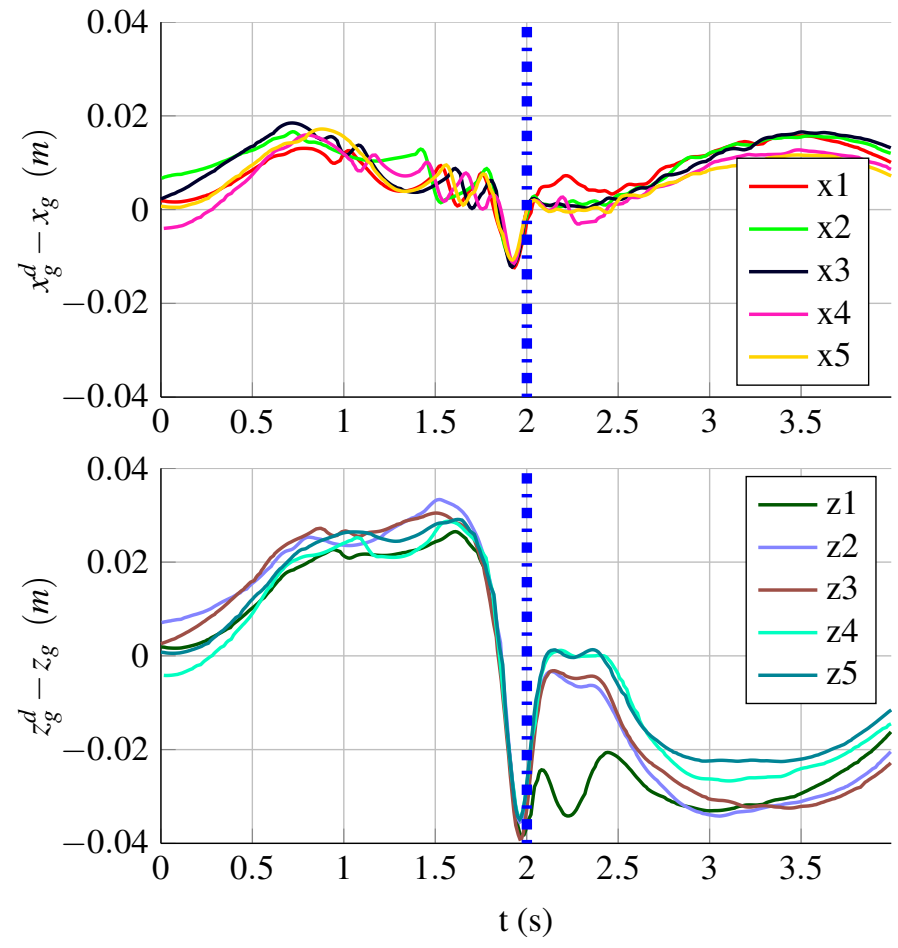

FIGURE 8. The $x$ and $z$ components of the normal error between the experimental and desired gripper positions for 5 consecutive trials. The actual pickup time is represented by a vertical dashed line.

TABLE 2. Units of Non-Dimensionalization Factors

\begin{tabular}{c||c|c} 
& $\begin{array}{c}\text { Eagle trajectory } \\
\text { from video } \\
\text { footage }\end{array}$ & $\begin{array}{c}\text { Robot trajectory } \\
\text { estimated } \\
\text { using VICON }\end{array}$ \\
\hline$L_{g}$ & pixels & meters \\
$v_{p}$ & pixels / frame & meters / second
\end{tabular}

the axis of rotation to the gripping surface of the claw. The units are detailed in Table 2. Results using this approach are presented in Figs. 9 and 10. It can be seen that the $x$-position of the gripper followed closely to that of the eagle's claws. The significant deviation in $z$-position following pick-up can be attributed to the limited range of motion of the arm of the quadrotor compared to that of the eagle. Furthermore, the nondimensionalized length of the quadrotor's arm is slightly less than that of the fully extended eagle's leg. If the body length was used as the characteristic length, the gripper arm has a nondimensionalized length of 0.34 compared to the eagle's leg at 0.37 . 


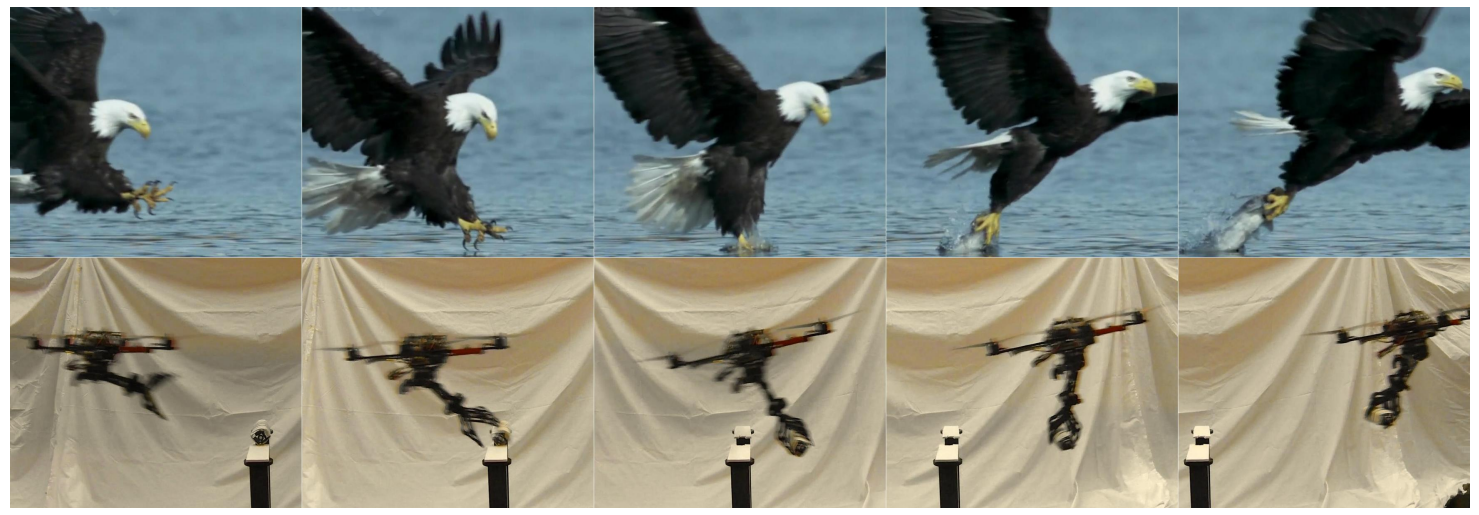

FIGURE 11. A still image comparison between the eagle (extracted from [4]) and the quadrotor for a trajectory with the quadrotor moving at $3 \mathrm{~m} / \mathrm{s}$ (9 body lengths / second) at pickup. See [21] for a video of the grasping.

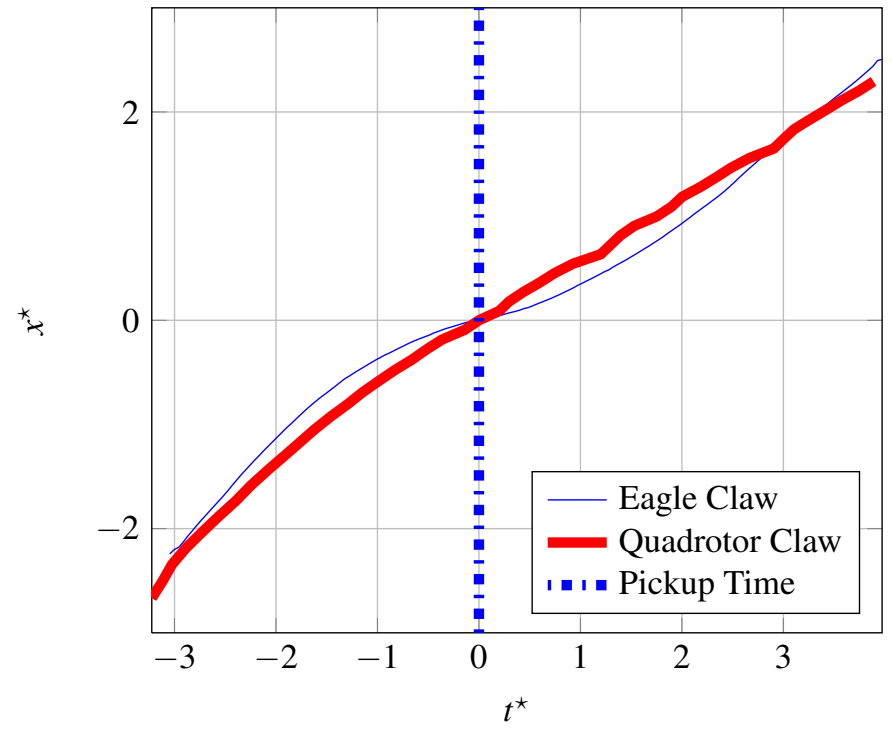

FIGURE 9. A comparison of the nondimensionalized $x$ positions of the quadrotor claw and the eagle claw. The lowest point on $z$ is considered to be the pickup point and is denoted by the vertical line. The eagle claw has a slower relative velocity at pickup than our claw when the quadrotor body speed is $2 \mathrm{~m} / \mathrm{s}$.

\section{CONCLUSION AND FUTURE WORK}

In this paper, we explored the challenges of high-speed aerial grasping using a quadrotor MAV equipped with a gripper. A novel appendage design, inspired by the articulation of an eagle's legs and claws, was shown to enable a high rate of success while grasping objects at high velocities. The dynamic model of the quadrotor and gripper system was shown to be differentially flat, and minimum snap trajectories were generated for dynamic grasping. Experimental results were presented for quadrotor velocities of $2 \mathrm{~m} / \mathrm{s}$ and $3 \mathrm{~m} / \mathrm{s}$ (6 - 9 body lengths / second). Finally,

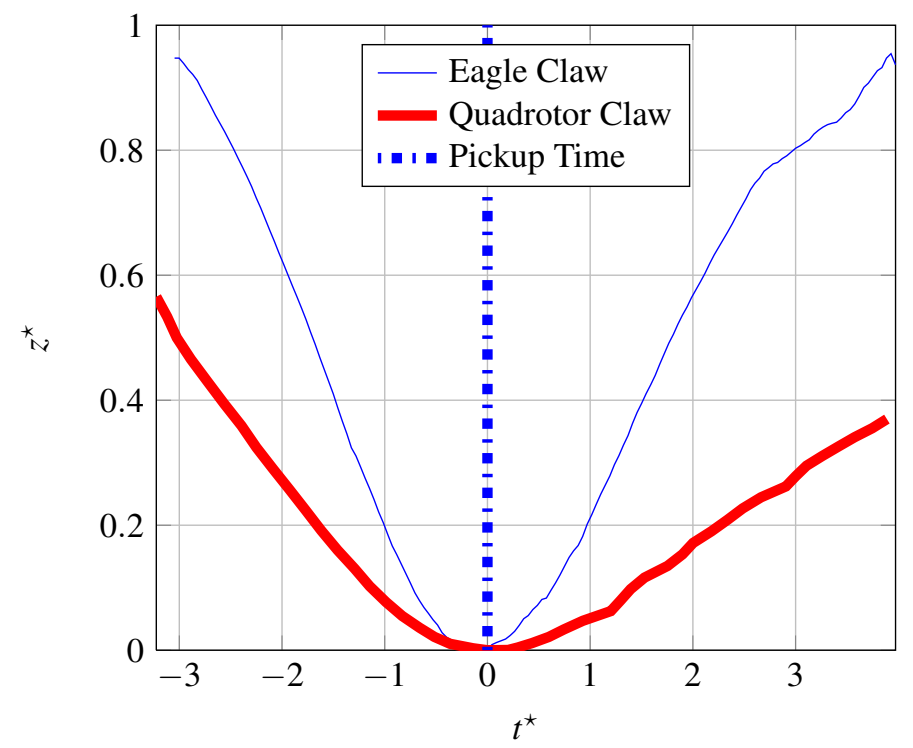

FIGURE 10. A comparison of the nondimensionalized $z$ positions of the quadrotor and the eagle claw. The vertical line indicates the pickup time.

a comparison of a nondimensionalized quadrotor trajectory with a sample avian trajectory was presented.

Next, in regard to future research, we present three topics. First, just as an eagle is able to navigate based only on its own visual and inertial sensors, a quadrotor should be able to make in-flight corrections using data from an on-board camera. Towards this, we aim to accomplish high-speed aerial grasping of outdoor objects through control based on visual servoing, rather than through a VICON motion capture system. A second direction of research is motivated by the fact that, for energy efficiency and stealth, resting on a stationary fixture is preferable to hover- 
ing. Thus, we aim to achieve autonomous detection of candidate sites and corresponding controlled landing for perching at these sites. Finally, we will pursue novel manufacturing methods to create appendages with lower inertia, which will enable more agile grasping and perching strategies.

\section{REFERENCES}

[1] Lindsey, Q., Mellinger, D., and Kumar, V., 2011. "Construction of Cubic Structures with Quadrotor Teams". In Robotics: Science and Systems.

[2] Sreenath, K., Michael, N., and Kumar, V., 2013. "Trajectory Generation and Control of a Quadrotor with a CableSuspended Load A Differentially-Flat Hybrid System”. In International Conference on Robotics and Automation.

[3] Mellinger, D., Shomin, M., Michael, N., and Kumar, V., 2010. "Cooperative grasping and transport using multiple quadrotors". In International Symposium on Distributed Autonomous Robotic Systems.

[4] Bass, K., Leith, B., Anderson, J., Bassett, P., Stevens, J., Pearson, H., and Turner, J., 2009. Nature's Most Amazing Events. [DVD]. BBC Worldwide Ltd. Programs.

[5] Venable, N., 1996. Birds of Prey. West Virginia University Extension Service.

[6] Mellinger, D., Michael, N., and Kumar, V., 2010. "Trajectory Generation and Control for Precise Aggressive Maneuvers with Quadrotors". In International Symposium on Experimental Robotics.

[7] Kumar, V., and Michael, N., 2012. "Opportunities and challenges with autonomous micro aerial vehicles". The International Journal of Robotics Research, 31(11), Aug., pp. 1279-1291.

[8] Dollar, A. M., and Howe, R. D., 2010. “The Highly Adaptive SDM Hand: Design and Performance Evaluation". The International Journal of Robotics Research, 29(5), Feb., pp. 585-597.

[9] Doyle, C. E., Bird, J. J., Isom, T. A., Johnson, C. J., Kallman, J. C., Simpson, J. A., King, R. J., Abbott, J. J., and Minor, M. A., 2011. "Avian-inspired passive perching mechanism for robotic rotorcraft". In 2011 IEEE/RSJ International Conference on Intelligent Robots and Systems, IEEE, pp. 4975-4980.

[10] Mellinger, D., Lindsey, Q., Shomin, M., and Kumar, V., 2011. "Design, modeling, estimation and control for aerial grasping and manipulation". In 2011 IEEE/RSJ International Conference on Intelligent Robots and Systems, IEEE, pp. 2668-2673.

[11] Mellinger, D., Shomin, M., and Kumar, V., 2010. "Control of Quadrotors for Robust Perching and Landing". In International Powered Lift Conference.

[12] "Festo Coorporate," Accessed April 18, 2013, ExoHand, from http://www.festo.com/cms/en_corp/12713.htm.
[13] Dollar, A. M., and Howe, R. D., 2011. "Joint coupling design of underactuated hands for unstructured environments". The International Journal of Robotics Research, 30(9), June, pp. 1157-1169.

[14] Dollar, A., and Howe, R., 2006. "A robust compliant grasper via shape deposition manufacturing". IEEE/ASME Transactions on Mechatronics, 11(2), Apr., pp. 154-161.

[15] Michael, N., Mellinger, D., Lindsey, Q., and Kumar, V., 2010. "The GRASP multiple micro-UAV testbed". Robotics \& Automation Magazine, IEEE, 17(3), pp. 5665.

[16] Mellinger, D., and Kumar, V., 2011. "Minimum snap trajectory generation and control for quadrotors". In 2011 IEEE International Conference on Robotics and Automation, IEEE, pp. 2520-2525.

[17] Fliess, M., Levine, J., Martin, P., and Rouchon, P., 1995. "Flatness and defect of non-linear systems: introductory theory and examples". International Journal of Control, 61(6), June, pp. 1327-1361.

[18] Murray, R., Rathinam, M., and Sluis, W., 1995. "Differential flatness of mechanical control systems: A catalog of prototype systems". In ASME International Congress and Exposition, Citeseer.

[19] "Vicon Motion Systems, Inc," http://www.vicon.com.

[20] "Ascending Technologies, GmbH," http://www.asctec.de.

[21] Thomas, J., Polin, J., Sreenath, K., and Kumar, V., 2013. February 7, 2013, Avian-Inspired Grasping for Quadrotor Micro UAVs, from https://www.youtube.com/watch?v=ol8c9bdp7YI.

\section{APPENDIX: DIFFERENTIAL FLATNESS}

A key property of the coupled system, comprising of the quadrotor and actuated gripper, that was used for dynamic trajectory generation is differential flatness. Here we establish that the the coupled system is differentially flat, and that a set of flat outputs are given by (9). To do this, we first define $m_{s}=m_{q}+m_{g}$ so that the center of mass of the coupled system is

$$
\mathbf{r}_{s}=\frac{m_{q} \mathbf{r}_{q}+m_{g} \mathbf{r}_{g}}{m_{s}}
$$

We recall from (1) that $\mathbf{r}_{q}=\mathbf{r}_{q}\left(x_{q}, z_{q}\right)$ and $\mathbf{r}_{g}=\mathbf{r}_{g}\left(x_{q}, z_{q}, \beta\right)$. Thus, $\mathbf{r}_{s}=\mathbf{r}_{s}\left(x_{q}, z_{q}, \beta\right)$ and $\ddot{\mathbf{r}}_{s}$ is fully defined using the proposed flat outputs and their derivatives. In addition, this motivates the choice of $\beta$ instead of $\gamma$ defining the angle of the gripper. If the gripper angle was defined relative to the quadrotor attitude, then $\theta$ would appear in $\mathbf{r}_{s}$ and we would ultimately see that $\gamma$ is not a flat output. Further, it makes sense to plan using $\beta$ because at pickup, we have strict position constraints on the end effector that must be invariant to the attitude of the quadrotor. Defining 
$\mathbf{e}_{3}$ as the third standard basis vector, the Newton-Euler equations of motion provide

$$
m_{s} \ddot{\mathbf{s}}_{s}=u_{1} \mathbf{b}_{3}-m_{s} g \mathbf{e}_{3}
$$

revealing that

$$
u_{1}=m_{s}\left\|\ddot{\mathbf{r}}_{s}+g \mathbf{e}_{3}\right\|
$$

and

$$
\mathbf{b}_{3}=\frac{\ddot{\mathbf{r}}_{s}+g \mathbf{e}_{3}}{\left\|\ddot{\mathbf{r}}_{s}+g \mathbf{e}_{3}\right\|}
$$

from which $\theta$ can be determined. In addition, (18) requires that $\left\|\ddot{\mathbf{r}}_{s}+g \mathbf{e}_{3}\right\|>0$ or that $u_{1}>0$. Since the system is restricted to the planar case, $\mathbf{b}_{2}=\mathbf{e}_{2}$ and $\mathbf{b}_{1}=\mathbf{b}_{2} \times \mathbf{b}_{3}$. Next, we differentiate (16) to obtain

$$
m_{s} \dddot{\mathbf{r}}_{s}=\dot{u}_{1} \mathbf{b}_{3}+\Omega \times u_{1} \mathbf{b}_{3}
$$

where $\Omega=\dot{\theta} \mathbf{b}_{2}$. The projection onto $\mathbf{b}_{3}$ reveals

$$
\dot{u}_{1}=\mathbf{b}_{3} \cdot m_{s} \dddot{\mathbf{r}}_{s}
$$

and, using this relationship,

$$
\Omega \times \mathbf{b}_{3}=\frac{m_{s}}{u_{1}}\left(\dddot{\mathbf{r}}_{s}-\left(\mathbf{b}_{3} \cdot \dddot{\mathbf{r}}_{3}\right) \mathbf{b}_{3}\right)
$$

We notice that this is purely in the $\mathbf{b}_{1}-\mathbf{b}_{2}$ plane and, more specifically, that $\Omega \times \mathbf{b}_{3}=\dot{\theta} \mathbf{b}_{1}$ Thus,

$$
\dot{\theta}=\frac{m_{s}}{u_{1}}\left(\mathbf{b}_{1} \cdot \dddot{\mathbf{r}}_{s}\right)
$$

Next, we take the second derivative of (16) to obtain

$$
\begin{aligned}
m_{s} \mathbf{r}_{s}^{(4)}=\dot{u}_{1} \dot{\theta} \mathbf{b}_{2} \times & \mathbf{b}_{3}+\ddot{u}_{1} \mathbf{b}_{3}+\dot{\theta} \mathbf{b}_{2} \times \dot{u}_{1} \mathbf{b}_{3} \\
& +\dot{\theta} \mathbf{b}_{2} \times\left(\dot{\theta} \mathbf{b}_{2} \times u_{1} \mathbf{b}_{3}\right)+\ddot{\theta} \mathbf{b}_{2} \times u_{1} \mathbf{b}_{3} .
\end{aligned}
$$

Collecting terms and simplifying cross products,

$$
m_{s} \mathbf{r}_{s}^{(4)}=\left(2 \dot{u}_{1} \dot{\theta}+\ddot{\theta} u_{1}\right) \mathbf{b}_{1}+\left(\ddot{u}_{1}-\dot{\theta}^{2} u_{1}\right) \mathbf{b}_{3} .
$$

The projections onto $\mathbf{b}_{1}$ and $\mathbf{b}_{3}$ reveal

$$
\ddot{\theta}=\frac{1}{u_{1}}\left(m_{s} \mathbf{b}_{1} \cdot \mathbf{r}_{s}^{(4)}-2 \dot{u}_{1} \dot{\theta}\right)
$$

and

$$
\ddot{u}_{1}=\mathbf{b}_{3} \cdot\left(m_{s} \mathbf{r}_{s}^{(4)}\right)+\dot{\theta}^{2} u_{1} .
$$

Next, we let $F_{x}$ and $F_{z}$ be reaction forces at the attachment point of the gripper so that the translational and angular equations of motion of the gripper are

$$
\begin{aligned}
m_{g} \ddot{x}_{g} & =F_{x} \\
m_{g} \ddot{z}_{g} & =F_{z}-m_{g} g \\
J_{g} \ddot{\beta} & =\tau+F_{x} L_{g} \sin (\beta)+F_{z} \cos (\beta) .
\end{aligned}
$$

Solving for the gripper arm actuator torque, $\tau$,

$$
\tau=J_{g} \ddot{\beta}-L_{g} m_{g}\left(\ddot{x}_{g} \sin (\beta)+\left(\ddot{z}_{g}+g\right) \cos (\beta)\right) .
$$

Lastly, we know that

$$
u_{3}=\ddot{\theta} J_{q}+\tau
$$

Thus, we have demonstrated that the state and the inputs of the coupled system are functions of the flat outputs and their derivatives, provided that they are sufficiently smooth, which establishes that the system is differentially flat. 